\title{
DEVELOPMENT OF A PAPER-BASED APPROACH FOR THE DETECTION OF CARBONYL COMPOUNDS IN INDOOR AIR SAMPLES
}

Madavie M.G.Y1 and Kaumal N. M.1*

1 Department of Chemistry, University of Colombo, Colombo 03

DOl: http://doi.org/10.4038/sljb.v2i2.8

\begin{abstract}
This study contributes to the development of a method to measure the level of airborne carbonyl compounds. Carbonyl compounds (CC) are very common indoor air pollutants which are widely used in building and home furnishing industries. Most CC off-gas or vaporize even at normal room temperatures, which results in an increase in the indoor volatile organic carbon levels. Acute and chronic exposure to formaldehyde can result in respiratory symptoms and eye, nose, and throat irritation in humans. Because of these adverse health effects and the increased levels of $\mathrm{CC}$ in indoor air and residential areas, there has been a considerable attention toward the development of sensitive methods to monitor indoor CC levels. Most methods available for monitoring indoor CC levels are indirect and time consuming. This paper describes a novel, portable, on site, low-cost and green method for regular monitoring of $\mathrm{CC}$ in air.

In this method, the derivatization reagent, 2,4-dinitrophenylhydrazine (2,4-DNPH) was used to capture the airborne CC. Formaldehyde was used as the CC for this study. A novel paper-based platform soaked with 2,4 DNPH was used for the quantification of air born CC. Derivatives of CC were formed on a paper-based platform which were then directly analyzed using FTIR or an image scanner. The FTIR response for the carbonyl derivatives, which correlates with the levels of airborne CC, was used to quantify the levels of airborne CC. The same was scanned using a high resolution image scanner to obtain the RGB values and the yellow color intensity was calculated. Based on the RGB values, the produced yellow color intensity and the levels of CC produced a linear relationship. The use of an image scanner to quantify the levels of CC makes this method a portable, sensitive and on-site CC detection method. This newly developed paperbased FTIR method is agreeable with the stranded HPLC method at 95\% confidence limit.
\end{abstract}

Key words: Air born carbonyl compounds, paper-based tool, FTIR, portable tool

*Corresponding author: E-mail: mnkaumal@sci.cmb.ac.lk

(iD) http://orcid.org/0000-0001-8506-8431 


\section{Introduction}

Analysis of levels of volatile organic compounds (VOC) emitted to the environment has gained special attention within past few decades due to their potential impact on human health. Carbonyl compounds (CC) can be commonly associated with various processes produce adverse conditions to humans (Yu et al., 2011). Because of the potential impact on human health, it is important to monitor the levels of CC in enclosed areas used for human occupation. The most common CC that can be found in enclosed areas include formaldehyde, acetaldehyde, acrolein and acetone. Many man-made commonly found utilities and some human activities are responsible for the emission of CC to the environment. Out of the many manmade utilities, house hold furniture, electronic products, plastic-based products and recycled product are the main sources of CC (Weng et al., 2010). Formaldehyde is the most common CC found in residential indoor air. Even though the method reported can detect the total CC present in indoor air, only formaldehyde is used in the process of method development and validation in this study.

Most commonly used method for the collection of airborne CC is to use an adsorbing material with a derivatization agent to capture CC, followed by solvent (Soman et al., 2008) or thermal desorption (Ho, et al., 2004) and analysis using a chromatographic or spectroscopic methods (Szulejko, et al., 2015). Various derivatization materials are reported by many research groups (Szulejko et al., 2015). However 2,4-di-nitrophenyl hydrazine (2,4-DNPH) is the most commonly used derivatization agent
(Szulejko et al., 2015). Even though there are few drawbacks (Pal et al., 2007), the 2,4-DNPH derivatization-based HPLC method can be considered as a cheaper and easier methods for the determination of CC. However, the use of advanced instruments such as HPLC-UV/VIS and GC/MS methods are not suitable to monitor the onsite CC levels under nonlaboratory conditions. In addition to the chromatographic method (Ho et al., 2004), electrochemical sensors (Otson et al., 1988) are also available for the detection of some CC. However, the lack of sensitivity and selectivity limits the usability of these sensors.

Paper-based analytical tools are popular among scientists due to their simplicity, low cost, environmental friendly nature and portability. Development of paperbased tools for the detection of alcohol is previously reported (Gunawardhana et al., 2016). Objective of this study is to develop a simple and green paper-based tool that can be used as an onsite method to detect CC. A paper-based tool capable of detecting CC with minimum amount of organic solvents was successfully developed under this study.

\section{Material and Methods}

\subsection{Chemicals and other materials}

All chemicals were used as they were received unless specified. Conc. hydrochloric acid (37\%), 2,4-DNPH and methanol were purchased from Sigma, Germany. Acetonitrile and formaldehyde were purchased from Merck Millipore.

\subsection{Reagents}

A 2,4-DNPH solution was prepared by mixing $0.01 \mathrm{~g}$ of 2,4-DNPH with $100 \mu \mathrm{L}$ of 
$\mathrm{HCl}$ and diluting up to $100.00 \mathrm{~mL}$ using distilled methanol. A series of formaldehyde standards with the concentrations of $1,5,10,15$, and $20 \mathrm{ppm}$ was prepared using the $100.0 \mathrm{ppm}$ standards of formaldehyde in methanol solution.

The calibration standard for HPLC analysis was prepared by mixing a solution of 2,4-DNPH (1.00 g of 2,4-DNPH $+2.00 \mathrm{~mL}$ of conc. $\mathrm{HCl}$ diluted up to $100.00 \mathrm{~mL}$ using distilled methanol) with excess acetone. The resultant precipitate was re-crystallized to obtain pure formaldehyde 2, 4-DNPH (HCHO-DNPH) derivatives. A series of standard solutions with the concentrations of 1,5 , $10,15,20 \mathrm{ppm}$ were prepared using the purified HCHO-DNPH derivative using methanol as the solvent.

\subsection{Instruments}

An FTIR Spectrophotometer (Varian 660IR) was used to measure the absorbance and percentage transmittance (\%transmittance) of HCHO-DNPH complex. High performance liquid chromatography (System Controller: Jasco 802-Sc with UV/VIS detector), was used for method validation. An HP image scanner (HP Scan jet 2400) was used to obtain the high resolution image from the paper based devices, to determine the yellow color intensity (yellow number) developed region.

\subsection{Development of paper-based devices}

Disk shaped $($ diameter $=0.7 \mathrm{~cm})$ devices were developed using Whatman No 1 filter papers. These disk shaped devices were soaked with 2,4-DNPH solution and dried to develop the paper-based tool. Blank response was recorded using devices soaked only with the 2,4-DNPH solution.

\subsection{Construction of calibration plot using FTIR measurement}

Disk shaped $($ diameter $=0.7 \mathrm{~cm})$ devices were developed using Whatman No 1 filter papers and soaked with standard solutions of HCHO-DNPH derivative in methanol. These disks were dried and the $\%$ transmittance at $1648 \mathrm{~cm}^{-1}$ was recorded using the FTIR. The lowest \%transmittance observed around 1648 $\mathrm{cm}^{-1}$ for each disk soaked with standard solutions of HCHO-DNPH derivative was used to construct the calibration plot.

\subsection{Construction of calibration plot using high resolution image scanner}

Disk shaped $($ diameter $=0.7 \mathrm{~cm})$ devices were developed using Whatman No 1 filter papers and soaked with standard solutions of HCHO-DNPH derivative in methanol. Then the paper discs were scanned with an image scanner (HP Scan jet 2400) and then imported to Adobe Photoshop CS5®. Use of an image scanner to measure the colour intensity of paperbased devices is reported previously (Liana et al., 2012). These images were converted to grayscale mode and the yellow color intensity was computed using the three primary colors. A graphical user interface (GUI) was developed to select individual paperbased device when multiple disks were scanned simultaneously. The averages of red, green and blue color intensities over a range of 50 pixels on the paper-based tool were taken for better accuracy. The center of the paper-based device was selected by using the GUI and the average yellow color intensity was calculated over a circular area with a diameter of 50 
pixels. The calibration curve was then plotted between the mean color intensity versus the concentration.

\subsection{Construction of calibration plot using HPLC method}

A method reported by Soman et. al. (2008) was used for the quantification of HCHO-DNPH derivative. Double distilled acetonitrile and water 40:60 mixture was used as the mobile phase. A volume of 20 $\mu \mathrm{L}$ from each standard solutions of HCHODNPH derivative in methanol was analyzed. The peak area for each standard solution was used to construct the calibration plot.

\subsection{Method validation}

A gaseous phase formaldehyde series with concentrations of $0.2,0.4,0.6,0.8,1$ $\mathrm{mg} / \mathrm{L}$ was prepared in a glass container of 5 L by introducing the standard formaldehyde solution. The air borne formaldehyde concentration in glass containers was estimated using the volume of glass container and the amount of formaldehyde introduced. Glass container was kept tightly closed for six hours after introducing the paper-based tool soaked with 2,4-DNPH. After six hours, paper-based devices were analyzed using FTIR and image scanner method. A second set of devices exposed to the standard formaldehyde air samples for a period of six hours were extracted in to a solution of methanol and the methanol extract was analyzed using HPLC (Pal et al., 2007).

\subsection{Analysis of real air samples}

An enclosed air tight room space contaminated with formaldehyde was tested using the paper-based devices and
HPLC (Pal,et al., 2007) and FTIR methods were used to quantify the levels of CC in the air tight room. Two methods were compared statistically at $95 \%$ confidence limit using the method explained in Skoog et al.

\section{Results}

Paper-based devices soaked with the 2,4DNPH solutions and treated with standard formaldehyde solutions were scanned with FTIR produced a characteristic transmittance pattern for the HCHO-DNPH derivative. The FTIR response for a 2,4-DNPH soaked paperbased device exposed to an air sample with formaldehyde is shown in Figure 1. The expected unique \%transmittance for HCHO-DNPH was observed around 1648 $\mathrm{cm}^{-1}$ due to stretching vibrations of the double bond between $\mathrm{C}$ and $\mathrm{N}(\mathrm{C}=\mathrm{N})$. The calibration plot constructed based on FTIR response is shown in Figure 2. The calibration plot developed based on HPLC study is shown in Figure 3.

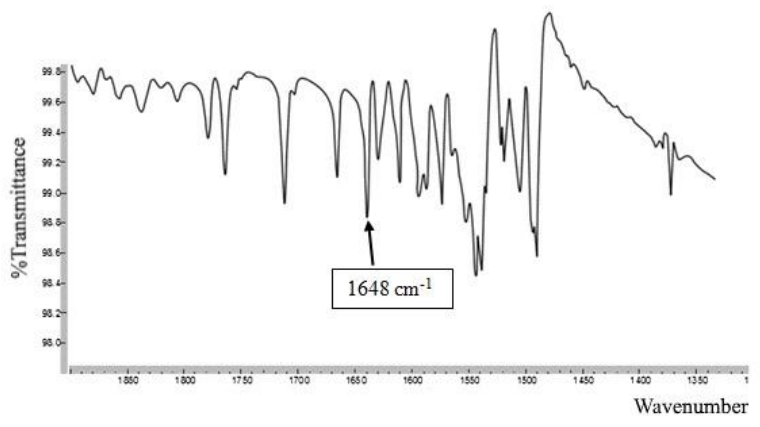

Figure 1. FTIR response for a 2,4-DNPH soaked paper-based device exposed to an air sample with formaldehyde.

Paper-based devices exposed to air samples with known formaldehyde concentrations were analyzed using the 
FTIR and HPLC methods and the results are given in Table 1.

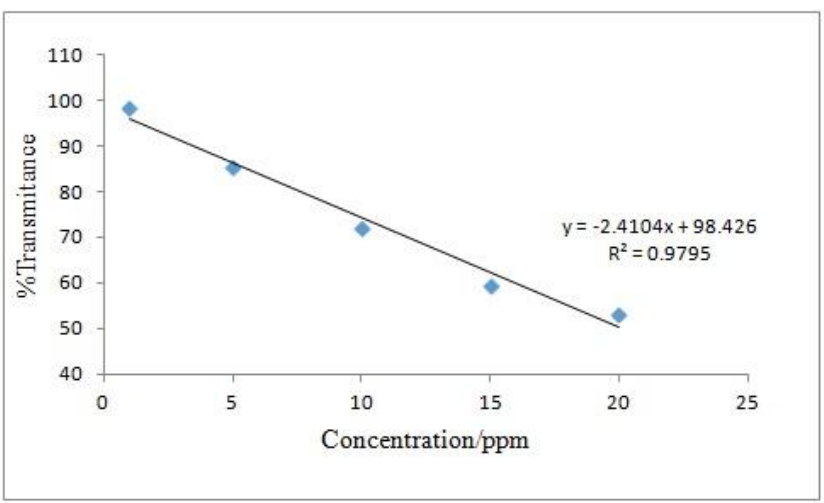

Figure 2. Calibration plot of FTIR \%transmittance against formaldehyde 2,4-DNPH derivative concentration.
Indoor air tested of enclosed areas contaminated with five various levels of

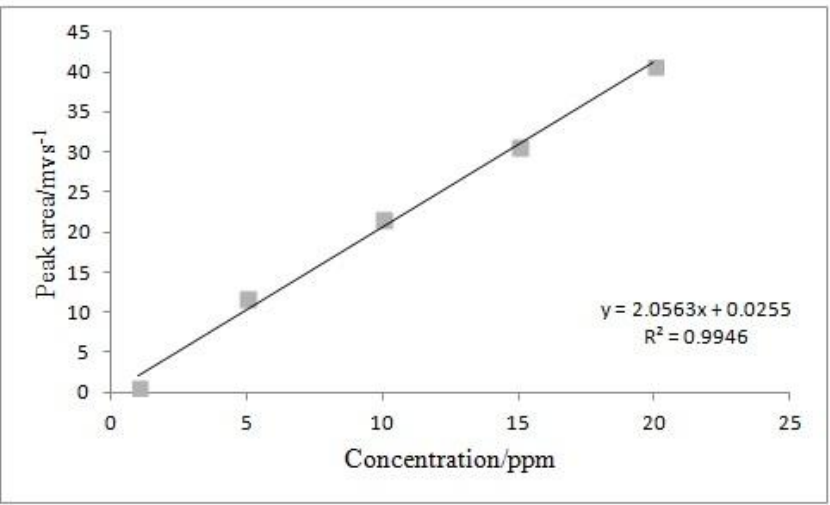

Figure 3. Calibration plot of peak area of HPLC response against standard formaldehyde 2,4-DNPH derivative concentration.

Table 1. Response of the paper-based tool exposed to air samples with the formaldehyde concentrations of $0.2,0.4,0.6,0.8,1 \mathrm{mg} / \mathrm{L}$ were analyzed using the FTIR and HPLC methods.

\begin{tabular}{ccc}
\hline Concentration $/ \mathrm{mg} / \mathrm{L}$ & Peak area/mvs & \%Transmittance \\
\hline 0.2 & 0.91 & 97.65 \\
0.4 & 2.90 & 95.70 \\
0.6 & 4.41 & 93.90 \\
0.8 & 6.67 & 91.14 \\
1.0 & 8.14 & 89.63
\end{tabular}

The RBC values, yellow value and the intensities of each paper-based device soaked with 2,4-DNPH exposed to the known formaldehyde air samples are given in the Table 2. The correlation between yellow values against formaldehyde concentration of air samples is shown in Figure 6.
Indoor air tested of enclosed areas contaminated with five various levels of formaldehyde (sample number 01, 02, 03, 04 and 05) using the developed paperbased device and HPLC methods. Results obtain for these samples are given in Table 3. 


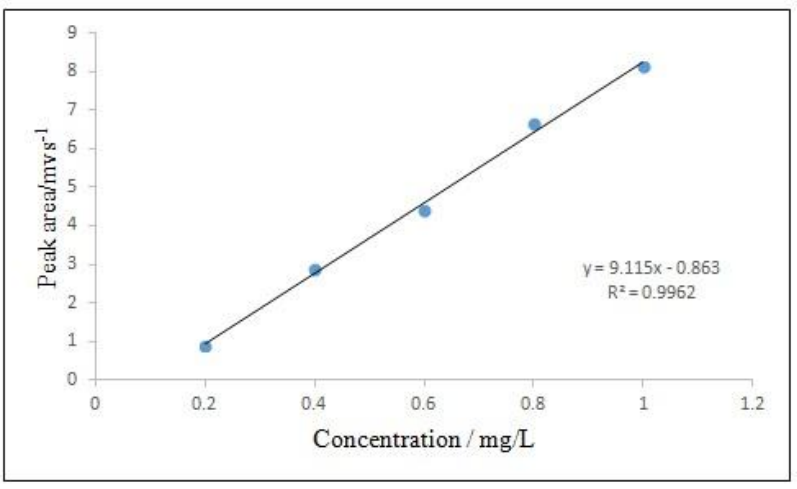

Figure 4. Correlation between the HPLC peak area and the formaldehyde concentrations of $0.2,0.4,0.6,0.8,1 \mathrm{mg} / \mathrm{L}$.

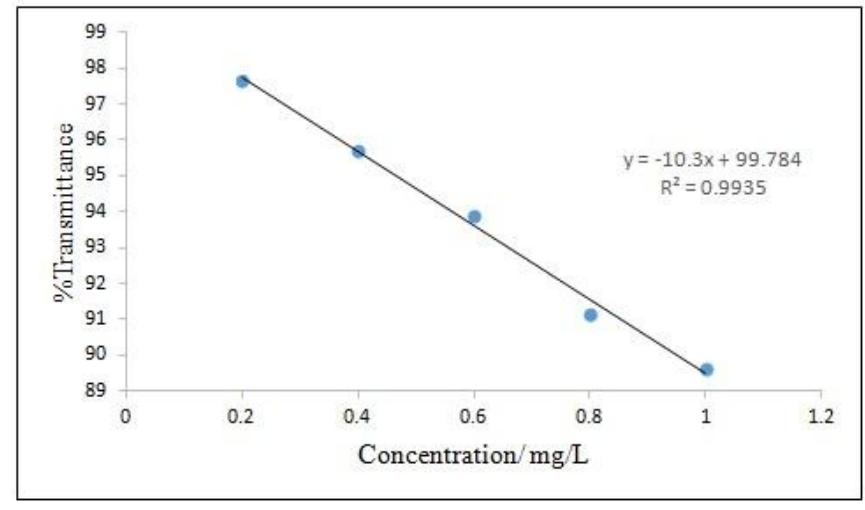

Figure 5. Correlation between the FTIR $\%$ transmittance and the formaldehyde concentrations of $0.2,0.4,0.6,0.8,1 \mathrm{mg} / \mathrm{L}$.

Table 2. The RGB values, yellow value and the intensities of each paper-based device exposed to $0.2,0.4,0.6,0.8,1 \mathrm{mg} / \mathrm{L}$ formaldehyde air samples 2,4-DNPH derivative concentrations

\begin{tabular}{cccccc}
\hline $\begin{array}{c}\text { Concentration/ } \\
\mathrm{mg} / \mathrm{L}\end{array}$ & Red & Green & Blue & Yellow & Intensity \\
\hline 0.2 & 245 & 242 & 42 & 211 & 244 \\
0.4 & 241 & 238 & 40 & 213 & 240 \\
0.6 & 245 & 241 & 37 & 216 & 243 \\
0.8 & 246 & 245 & 37 & 217 & 246 \\
1 & 217 & 206 & 31 & 219 & 212 \\
\hline
\end{tabular}

Table 3. The concentration calculated based on the HPLC method is corrected using the response obtained for the blank sample.

\begin{tabular}{rcc}
\hline $\begin{array}{l}\text { Sample } \\
\text { No }\end{array}$ & $\begin{array}{c}\text { Calculated concentration using } \\
\text { FTIR method / mg/L }\end{array}$ & $\begin{array}{c}\text { Calculated concentration using } \\
\text { standard HPLC method/ mg/L }\end{array}$ \\
\hline 1 & 0.05 & 0.09 \\
2 & 0.08 & 0.09 \\
3 & 0.25 & 0.35 \\
4 & 0.26 & 0.30 \\
5 & 0.27 & 0.30 \\
\hline
\end{tabular}




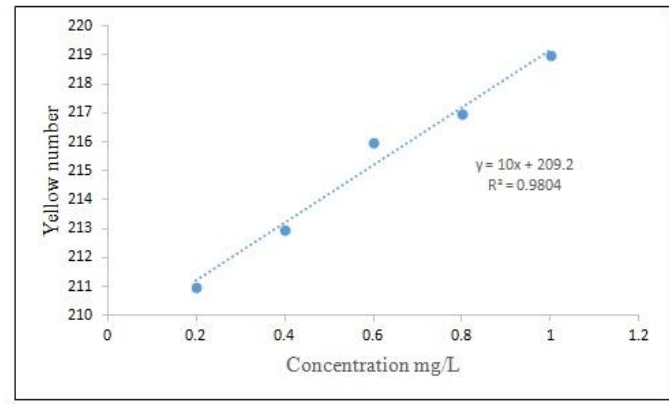

Figure 6. Correlation between the yellow value and formaldehyde concentrations of $0.2,0.4,0.6,0.8,1$ $\mathrm{mg} / \mathrm{L}$.

\section{Discussion}

The method described in this paper requires minute amounts of chemicals than that of other conventional methods used to quantify carbonyl compounds in indoor air. Hence, this novel method can be considered as a green technique for the determination of airborne total carbonyl compounds. Based on the repeatability and the correlation between $\%$ transmittance and formaldehyde concentration, disk shape was selected as the optimum shape for the paper-based device. Disk shape Whatman No 1 filter paper pieces soaked with 2,4-DNPH were used to trap CC. The chemical reaction between formaldehyde and 2,4-DNPH to from HCHO-DNPH derivatization is shown in Figure 7.
The resultant FTIR spectrum is shown in the Figure 01, and the peak generated due to the $\mathrm{N}-\mathrm{N}=\mathrm{C}$ can be seen around 1648 $\mathrm{cm}^{-1}$. The level of HCHO-DNPH derivatization product or any carbonyl compound-DNPH derivatives on the paper-based device is proportional to the $\%$ transmittance at $1648 \mathrm{~cm}^{-1}$. This correlation is used to produce a paperbased device that can be used to quantify the level of CC in air. Figure 2 shows the correlation between the \%transmittance and the levels of HCHO-DNPH derivative in the range of $1-20 \mathrm{ppm}$ on the paperbased device. The regression equation obtained for the paper-based FTIR detection was: $y=-2.4104 x+98.426$, where $y$ is the \%transmittance produced by the paper-based device and $x$ is the level of HCHO-DNPH derivative on the paper in ppm. The correlation coefficient of $0.9795(n=5)$ provides satisfactory linearity between the \%transmittance and the level of HCHO-DNPH derivative on the paper in the concentration range of 1 - 20 ppm. This study confirms the ability to use the proposed paper-based device for the detection of CC in air samples.

Paper-based devices soaked with 2,4DNPH were kept under air tight conditions until used. Some paper-based devices soaked with the 2,4-DNPH solution are shown in Figure 8 .<smiles>NNc1ccc([N+](=O)[O-])cc1[N+](=O)[O-]</smiles><smiles></smiles><smiles>C=NNc1ccc([N+](=O)[O-])cc1[N+](=O)[O-]</smiles>

Figure 7. The chemical reaction between formaldehyde and 2,4-DNPH. 

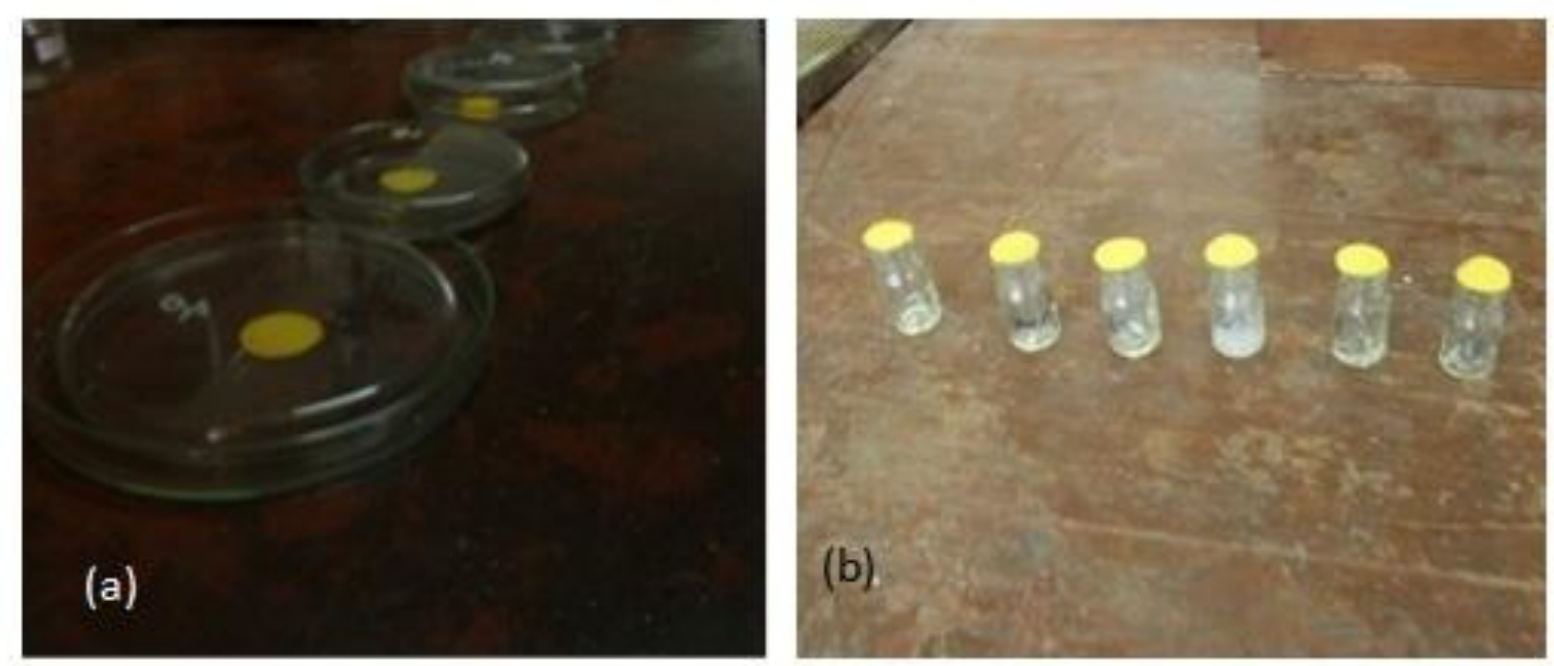

Figure 8. Paper based devices soaked with 2,4-DNPH solution (a): kept inside the Petri dishes and (b): kept on a small vial opening.

Previously reported HPLC based method was used to validate the novel method proposed based on a paper-based device to quantify the levels of CC in air. Figure 03 shows correlation between the area of the HPLC peak for the HCHO-DNPH derivative and the concentration in the range of $1-20 \mathrm{ppm}$. The regression equation obtained for the HPLC detection was: $y=2.0563 x+0.0255$, where $y$ is the peak area and $x$ is the concentration of HCHO-DNPH in ppm.

The proposed paper-based method was tested using laboratory prepared air samples with the concentrations of 0.2 , $0.4,0.6,0.8,1 \mathrm{mg} / \mathrm{L}$. These samples were prepared using $5 \mathrm{~L}$ glass bottles spiked with the standard formaldehyde volumes to build the required levels of formaldehyde. The paper-based devices were inserted in to the glass bottles and allowed to stand for a period of six hours.
The FTIR response produced for each paper-based device exposed to these laboratory prepared air samples are shown in Figure 5 . The regression equation obtained for the paper-based FTIR detection for standard formaldehyde air samples was: $y=-10.3 x$ +99.784 , where $y$ is the \%transmittance produce by the paper-based device and $x$ is the level of formaldehyde in $\mathrm{mg} / \mathrm{L}$. The correlation coefficient of $0.9935(n=5)$ provides satisfactory linearity between the \%transmittance and the level of formaldehyde in air samples in the concentration range of $0.2-1 \mathrm{mg} / \mathrm{L}$. Figure 04 shows the correlation between the area of the HPLC peak for the HCHODNPH derivative and with the concentrations of $0.2,0.4,0.6,0.8,1 \mathrm{mg} / \mathrm{L}$.

This proposed paper-based method does not require any mechanical method to collect air samples and the sample collection can be performed within six hours. The FTIR \%transmittance around 
$1648 \mathrm{~cm}^{-1}$ can be directly used for the quantification of CC. Based on Figure 5, the limit of detection(LOD) and the limit of quantification(LOQ) were calculated. Using the calibration plot shown in Figure 05, standard deviation of the slope (Sy) and the slope (S) were calculated. The LOD, which was calculated using the relationship $\mathrm{LOD}=3.3(\mathrm{Sy} / \mathrm{S})$, is 0.19 $\mathrm{mg} / \mathrm{L}$. The LOQ, which was calculated using the relationship, $\mathrm{LOD}=10(\mathrm{Sy} / \mathrm{S})$, is $0.29 \mathrm{mg} / \mathrm{L}$. The reported LOD and LOQ for the HPLC based detection of formaldehyde is 0.1 and $0.33 \mathrm{ppm}$, respectively (Soman et. al., 2008). Even though the proposed method is not capable of detecting lower concentrations as HPLC method, the proposed method is cheap, easy and uses less chemicals. LOD and LOQ of the proposed method can be improved by increasing the exposure time.

The method validation was done by testing an enclosed area contained with formaldehyde by using the new paperbased device and the standard HPLC methods. Five different concentrations (sample 1, 2, 3, 4 and 5) were set in the enclosed area and the CC levels were separately measured. The levels of CC obtained by both methods are given in Table 03. The two sets of experimental concentrations obtained using the new paper-based and HPLC methods were tested using the t-statistic according to the method given in Skoog et al. Based on the statistical evaluation, there is no significant difference at $95 \%$ confidence level between the two methods.

The proposed paper-based method is based on the FTIR detection and this hinders the ability to use this method without laboratory facilities. To overcome this drawback, the possibility of using a high resolution image scanner instead of FTIR detection was tested.

Paper-based devices exposed to laboratory prepared air samples were examined using a high resolution image scanner as explained in the Methodology section. Adobe Photoshop CS5 ® software was used to measure the yellow color intensity (yellow number) of the test zones of the paper-based microfluidic devices. All images were converted to grayscale mode in order to avoid unnecessary color detection by the software. Image scanner responses produced for each paper-based device exposed to laboratory prepared formaldehyde air samples are shown in Table 2 and Figure 6. The regression equation obtained for the paper-based image scanner detection for the standard formaldehyde air samples was: $y=10 x+$ 209.2, where $y$ is the yellow number generated for the paper-based device and $x$ is the level of formaldehyde in ppm. The correlation coefficient of $0.9804(n=5)$ provides satisfactory linearity between the yellow number and the level of formaldehyde in the air samples in the concentration range of $0.2-1 \mathrm{mg} / \mathrm{L}$. The LOD, which was calculated using the relationship, $\mathrm{LOD}=3.3(\mathrm{Sy} / \mathrm{S})$ is 0.07 $\mathrm{mg} / \mathrm{L}$. The image scanner-based method allows this proposed paper-based method to be used as a portable and user-friendly method. Also, this proposed method can be performed with laboratory facilities under industrial or domestic conditions to monitor the level of $\mathrm{CC}$ in any given situation.

Only limitation of this proposed method is that the paper based-device should be stored in an air tight container until use. Also, paper disks and 2,4-DNPH solution can be separately stored and the devices 
can be prepared when required. When an image scanner is used, the image can be digitally sent to a laboratory, so that the data can be analyzed by a specialist and the results can be sent real time, to the field. Recently, some research groups have managed to use smart phones to capture the color intensity of the paperbased device to quantify the level of analyte (Lee et al., 2011).The same approach can be used to improve this proposed paper-based device to detect CC. The amount of solvent and other chemicals used in both paper-based devices coupled FTIR and image scanner detection are lesser than the conventional HPLC method, hence these proposed methods can be considered as green methods.

\section{Conclusion}

This report presents the development and validation of a paper-based tool for the quantification of CC in indoor air. This method is an inexpensive, green, sensitive and reliable method for the detection of trace levels of CC. Circular shaped paperbased tools soaked with 2,4-DNPH successfully managed to capture airborne CC. The \%transmittance was measured using FTIR and the yellow color intensity was calculated using an image scanner. Percentage of transmittance around 1648 $\mathrm{cm}^{-1}$ and the yellow color intensity measured produced a linier correlation with the airborne formaldehyde concentration. An airtight region contaminated with formaldehyde was tested using the newly developed method and the standard HPLC method. Newly developed FTIR-based method is agreeable with the standards HPLC method at $95 \%$ confidence limit.

\section{References}

Gunawardhana, D.Y.R., Kaumal, M.N. (2016) Development of a portable paper-based microfluidic device for the detection of alcohol in biological fluids. Sri Lanka Journal of Biology, 1(1): 38-43.

Ho, Steven Sai Hang, Ho, K.F., Liu, W.D., Lee, S.C., Dai, W.T., Cao, J.J., Ip, H.S.S. (2011) Unsuitability of using the DNHP-coated solid sorbent cartridge for determination of airborne unsaturate carbonyls. Atmospheric Environment, 45: 261-265.

Ho, Steven Sai Hang, Yu, Jian Zhen (2004) Determination of airborne carbonyls: comparison of a thermal desorption/GC method with standard DNPH/HPLC method. Environmental Science \& Technology, 38(3): 862-870.

Lee, Dae-sik, Jeon, Byoung Goo, Ihm, Chunhwa, Park, Je-Kyun, Jung, Mun Yeon (2011) A simple and smart telemedicine device for developing regions: a pocket-sized colorimetric reader. Lab chip, 11: 120-126.

Levart, A., Veber, M. (2001) Determination of aldehydes and ketons in air samples using cryotrapping sampling. Chemosphere, 44: 701-708.

Lina, Devid D., Raguse, Burkhard, Justin Gooding, J. Chow, Edith (2012) Recent advances in paper-based sensors. Sensors, 12: 11505-11526.

Pal, R., Kim, K.H. (2007) Experimental choices for the determination of carbonyl compounds in air. Journal of separation science, $\mathbf{3 0}(\mathbf{1 6})$ : 2708-2718.

Pang, Xiaobing, Lewis, Alastair C., Hamilton, Jacqueline F. (2011) Determination of airborne carbonyls via pentafluorophenylhydrazine derivatization by GC-MS and its comparison with HPLC method, Talanta, 85: 206-414. 
Otson, R., Fellin, P. (1988) A review of techniques for measurement of airborne aldehydes. Science of total environment, 77: 95-131.

Skoog, Douglas A., West, Donald M., Holler, F. James, Crouch, Stanley R. (2014) Fundamentals of Analytical Chemistry, Brooks/Cole, USA, 136 p.

Soman, A., Qiu, Y., Li, Q. Chan (2008) HPLC-UV method development and validation for the determination of low level formaldehyde in a drug substance. Journal of Chromatographic Science. 46: 461-465.

Szulejko, Jan E., Kim, Ki-Hyun (2015) Derivatization techniques for determination of carbonyls in air. Trends in Analytical Chemistry, 64: 29-41.

Yu, Chuck W.F., Kim, Jeong Tai (2012) Longterm impact of formaldehyde and VOC emissions from wood-based products on indoor environments; and issues with recycled products. 21: 137-149. 